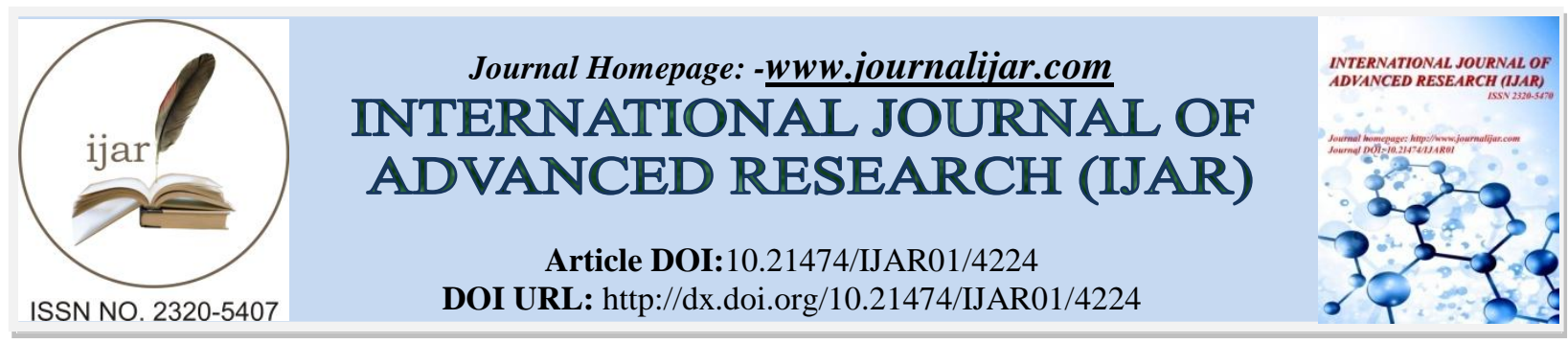

RESEARCH ARTICLE

\title{
BODY MASS INDEX ACCORDING TO INSULIN AND ORAL HYPOGLYCEMIC DRUG USAGE IN PATIENTS WITH TYPE 2 DIABETES.
}

Ammar Balkheyour ${ }^{1}$, Omar Ashour $^{1}$, Shahad AbuAlhamael ${ }^{2}$, Shima Aziz $^{3}$ and Hassan Alsini ${ }^{3}$.

1. Teaching assistant and Senior Registrar in internal medicine, King Abdulaziz University, Jeddah, Saudi Arabia.

2. Teaching assistant, Senior Registrar in internal medicine and Endocrine Fellow, King Abdulaziz University, Jeddah, Saudi Arabia.

3. Medical Resident, King Abdulaziz University Hospital, Jeddah, Saudi Arabia.

\section{Manuscript Info}

-.......................

Manuscript History

Received: 18 March 2017

Final Accepted: 21 April 2017

Published: May 2017

Key words:-

Diabetes, Obesity, Adults, Insulin.

\section{Abstract}

Objective: Obesity is highly prevalent in Saudi Arabia, resulting in an increase in the risk diabetes mellitus. A sedentary lifestyle with limited participation in outdoor activities could exacerbate the effects of obesity on diabetes mellitus. Moreover, although insulin replacement therapy is the most effective treatment for hyperglycemia in type 1 and advanced type 2 diabetes, it is associated with weight gain. Therefore, the aim of our study was to evaluate body mass index patterns, according to insulin and oral hypoglycemic drug (OHG) usage, in patients with type 2 diabetes in the western region of Saudi Arabia, who have a relatively sedentary lifestyle.

Methods: In this prospective study, 96 adults aged 40-90 years with type 2 diabetes were selected from among patients visiting the outpatient department of King Abdul Aziz University Hospital between February 1, 2017 and February 28, 2017. A majority of the participants were female $(68 \%)$. The body mass index of all study participants was measured and compared according to the presence of absence of insulin and OHG usage.

Results: Among the study participants, $75 \%$ and $33.3 \%$ were taking OHG and insulin, respectively. Evaluation of the patient BMI patterns revealed that patients $\mathrm{OHG}$ use had a significant negative correlation with increased BMI $(p=0.015)$. In addition, no significant difference in BMI was found between diabetic patients who were taking insulin and those not taking insulin ( $\mathrm{p}=0.093)$.

Conclusion: Our findings regarding the high prevalence of overweight or obesity among patients with diabetes suggests increased surveillance of weight in this patient population. Moreover, in diabetic patient in the western region of Saudi Arabia, insulin use plays a role in increasing weight, in addition to the limited outdoor activities and an unhealthy diet. Screening diabetes treatment outcomes and promoting awareness of the risk of obesity and diabetes and the effect of good lifestyle could improve the glycemic control and result in a good prognosis in patients with diabetes.

Copy Right, IJAR, 2017,. All rights reserved. 


\section{Introduction:-}

Diabetes Mellitus (DM) is one of the most common chronic diseases in the world and the most challenging health problems of the twenty-first century [1]. It is estimated that by the 2030 the number of people with diabetes will increase to more than 366 million [2, 3]. Most of these new cases are from developing countries, and the Middle East is estimated to be among the regions with the largest increase in the prevalence of diabetes by 2030 [3]. Five of the ten countries with the highest national prevalence of diabetes worldwide are in the Middle East [4]. Moreover, studies on DM prevalence in the gulf region have revealed variable but consistently high prevalence rates such as 25.7\% in Bahrain [5], 16.1\% in Oman [6], 18.7\% in the United Arab Emirates [4], and 14.6\% in Kuwait [4].Similarly, diabetes affects approximately $12 \%$ (26 million) of the US adult population, and is considered a twenty-first century epidemic [7]. The Kingdom of Saudi Arabia (KSA) is a rapidly developing country that faces the same threat; despite the KSA population having easy access to healthcare facilities, theprevalence of DM is $23.7 \%$, with nearly half of the diabetic population being newly diagnosed at the time of this study [8].

The prevalence of obesity, which is associated with the development of type 2 diabetes mellitus [9], has reached approximately $35.6 \%$ in the KSA, increasing in parallel with the increase in the prevalence of diabetes [10-12]. Moreover, approximately 45-65\% of patients with type 2 diabetes mellitus in the United States are obese [13]. The key risk factors for onset and poor clinical outcomes of diabetes include genetic predisposition, family history, overweight, obesity, hyperglycemia, hypertension, dyslipidemia, physical inactivity, ethnicity, and environmental factors [14].

Exogenous insulin replacement therapy remains the most effective treatment for hyperglycemia in type 1 and advanced type 2 diabetes, but in both cases, it regularly results in excessive weight gain that might adversely affect prognosis. [15] Therefore, the aim of this study was to evaluate the body mass index (BMI) of patients in Jeddah Saudi Arabia with type 2 diabetes according to insulin and oral hypoglycemic drug (OHG) therapy.

\section{Methodology:- \\ Patients:-}

Patients with type 2 diabetes who regularly attended the outpatient medical clinic at King Abdul-Aziz University Hospital between February 1, 2017 and February 28, 2017 were eligible for this study. The study inclusion criteria were as follows: age 40-90 years, age at diabetes onset $<20$ years, and duration of the disease $>2$ years. Exclusion criteria were advanced stages of chronic diabetes complications, additional comorbid diseases, or medications that could affect body weight. Patient information collected included name, age, sex, weight $(\mathrm{kg})$, height $(\mathrm{cm})$, and oral hypoglycemic medication or insulin use. Body weight was measured using a medical scale with an accuracy of 100 g. Body mass index (BMI) was calculated using the standard formula: weight $(\mathrm{kg}) / \mathrm{height}(\mathrm{m})^{2}$. Overweight was defined as a BMI of 25.0-29.9, and obesity as a BMI of $\geq 30.0$. Abdominal obesity was diagnosed according to International Diabetes Federation criteria [16]. The research ethics committee at King Abdul-Aziz University Hospital approved the study protocol.

\section{Statistical Analysis:-}

Data was entered, coded, cleaned, and analyzed using the statistical package for social science version 22 (IBM SPSS, Chicago, IL, USA). Assuming that the data followed normal distribution and excluding some continuous variable outliers, we performed statistical analysis to test the significance of differences in BMI between type 2 diabetic patients who were or were not taking insulin and those who were or were not taking OHG. Simple linear regression was used to determine the exact increase in BMI in $\mathrm{kg} \backslash \mathrm{m}^{2}$ in patients treated with OHG. In addition, simple descriptive statistics are reported as proportions for qualitative variables such as frequencies and percentages of patients treated with insulin and $\mathrm{OHG}$ also clarified BMI $\mathrm{kg} \backslash \mathrm{m}^{2}$ categories depends on exact value of BMI which is equal (weight $\mathrm{kg} \backslash$ height $\mathrm{m}^{2}$ ). Quantitative variablessuch as the patient age and BMI are reported as mean and standard deviation. The results were considered significant when $\mathrm{P}$-values were $<0.05$

\section{Results:-}

The study group comprised 96 patients (30 men and 66 women). Among the 96 patients enrolled in this study, 72 (75\%) were taking OHG and $24(25 \%)$ were not taking OHG (Figure. 1), and $32(33.3 \%)$ were taking insulin and 64 $(66.7 \%)$ were not taking insulin (Table. 1). Analysis of the distribution of patients across BMI categories revealed that 35 patients $(39.3 \%)$ were overweight, 27 patients $(30.3 \%)$ had class I obesity, 14 patients (15.7\%) class II obesity, and 13 patients had normal weight (14.6\%) (Figure. 2). The BMI of patients treated with OHG (30.13 \pm 4.7 
$\mathrm{Kg} / \mathrm{m}^{2}$ ) was significantly higher (by $2.8 \mathrm{~kg}^{2} \mathrm{~m}^{2}$ ) than that of patients not taking OHG $\left(27.35 \pm 3.4 \mathrm{Kg} / \mathrm{m}^{2}, \mathrm{p}=0.015\right)$. Moreover, no significant difference in BMI was found between diabetic patients taking insulin $(28.34 \pm 4.5 \mathrm{Kg} / \mathrm{m} 2)$ and those not taking insulin $(30.1 \pm 4.5 \mathrm{Kg} / \mathrm{m} 2, \mathrm{p}=0.093)$ (Figure. 3).

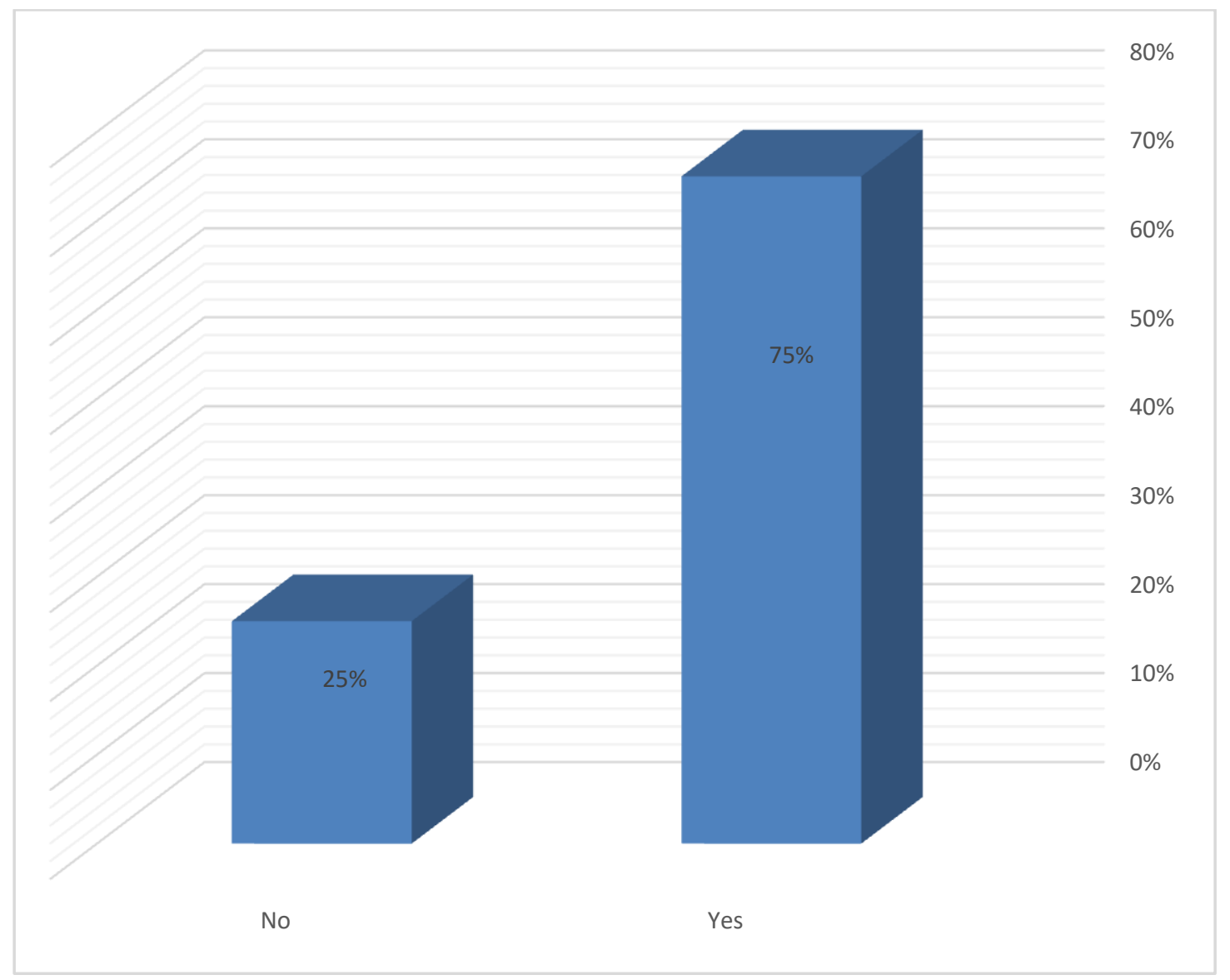

Figure 1:-Distribution of patients according to oral hyperglycemic treatment..

Table.1:- Distribution of patients according to insulin therapy.

\begin{tabular}{|c|c|c|}
\hline \multicolumn{2}{|c|}{ Insulin } \\
\hline Yes & Frequency & Percent \\
\hline No & 32 & 33.3 \\
\hline Total & 64 & 66.7 \\
\hline
\end{tabular}




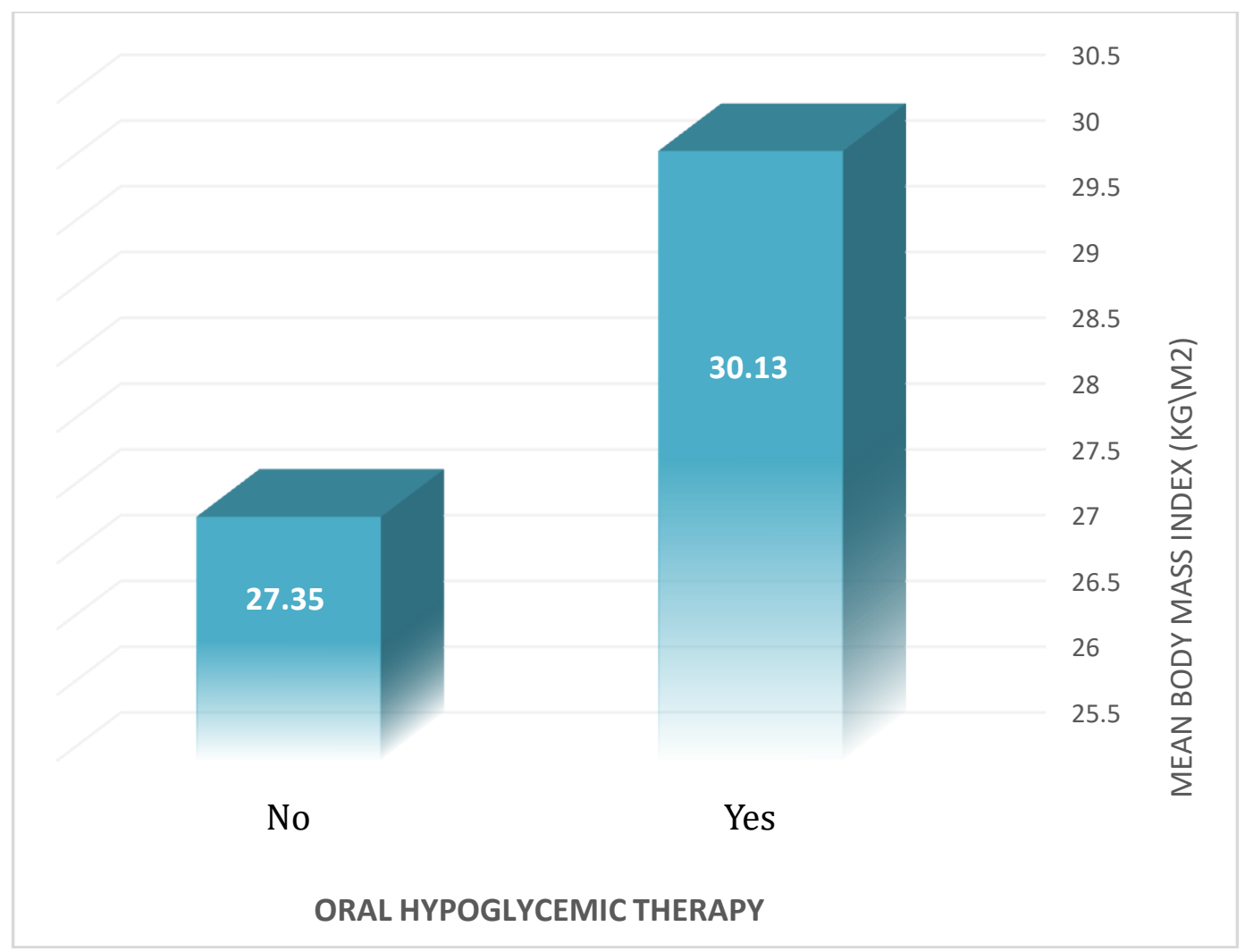

Figure 3:-BMI of patients is according to oral hypoglycemic therapy.

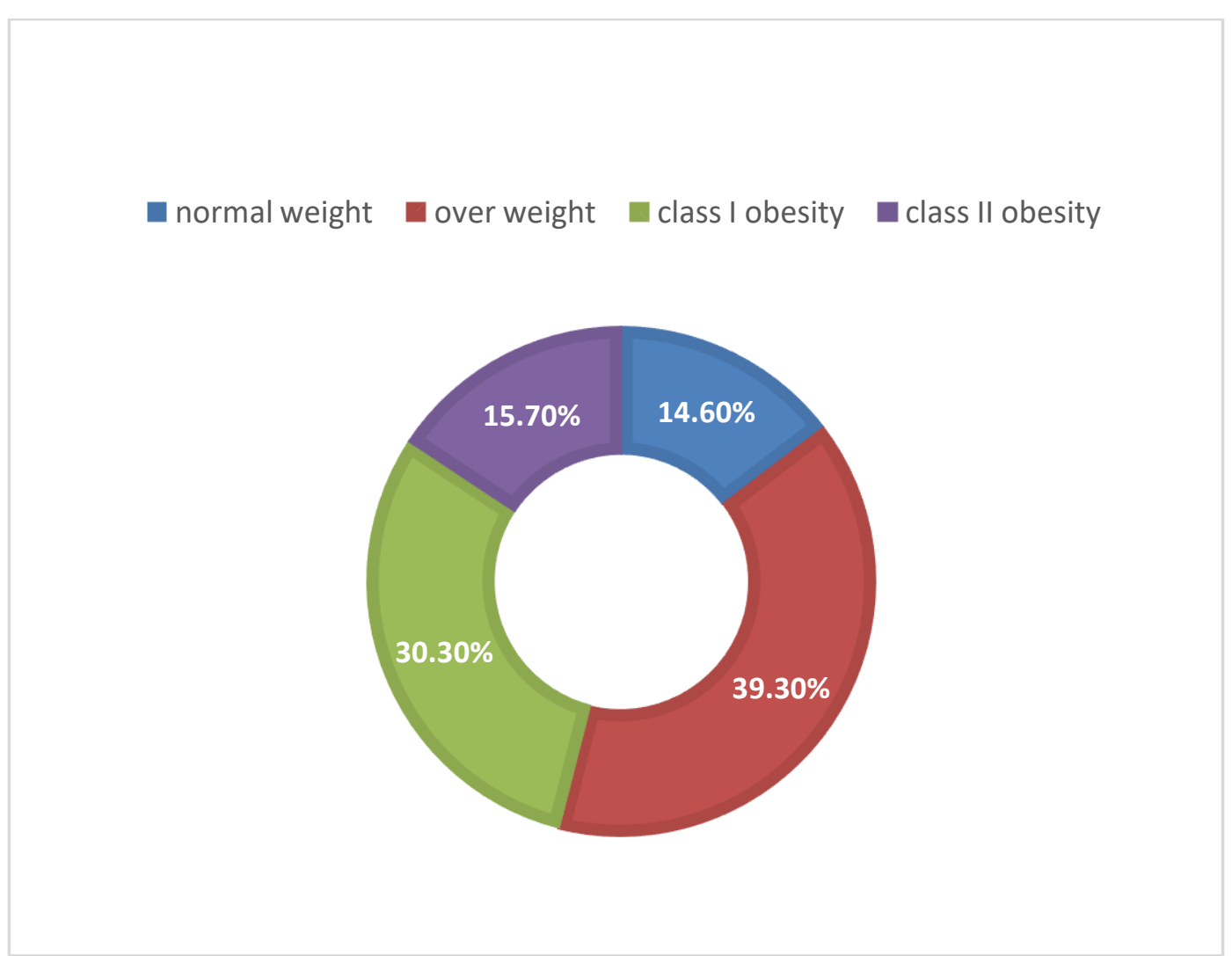

Figure 2:-Distribution of patients with type II diabetes across body mass index categories. 


\section{Discussion:-}

In the present study, we found that overweight individuals made up the largest proportion of patients with type 2 diabetes aged between 40 and 90 years. Insulin usage was not associated with any significant change in BMI in these patients but OHG usage was associated with a significantly higher BMI.

The higher prevalence of DM among our patient population is likely because only patients aged between 40 and 90 years were included, and diabetes prevalence is known to increase with age. Another possible reason is that risk factors for developing DM are also increasing in the KSA population, particularly, obesity and a sedentary lifestyle lacking physical activity. [8] In addition, female patients made up a majority of the study population. A previous Saudi Arabian study in 282 patients with diabetes aged 19-91 years (212 males and 70 females) revealed that female patients with diabetes had a significantly higher BMI than male patients with diabetes did $(p<0.001)$, which is consistent with previous findings in 1994 in the KSA [17]. This may be because most females in Saudi Arabia lead a sedentary indoor lifestyle with little exercise. Clothing might play a role in obesity as well; women in the Arab world often wear traditional long and wide dresses that can reduce their motivation to lose weight [18]. The etiology of obesity is far more complex than simply an imbalance between energy intake and energy output. Metabolic, endocrine, and genetic factors; dietary habits; physical activity; race; age; sex; drug use; and socioeconomic status all play a role in the development of obesity. These conventional factors along with several other factors such as increased access to private cars, employment of house cleaners, and a population-wide reduction in physical activity have contributed to the rise of obesity in the Arab world. The rise in caloric and fat intake in a region where exercise is not an integral part of the culture has added to the overall increased percentages of overweight and obese populations, but an increasingly westernized diet alone is not solely responsible for increased obesity in the Arab world. Eating food prepared outside the home is another serious contributing factor $[19,20]$. Obesity and particularly abdominal obesity are strongly associated with insulin resistance [21,22]. In turn, insulin resistance is an important risk factor for cardiovascular disease and increased morbidity and mortality in the general population [23]. However, insulin itself promotes weight gain, which in turn, increases cardiovascular risk [24, 25]. The underlying causes of insulin-induced weight gain include conservation of glucose calories that were previously utilized, and perhaps increased calorie intake as a defense against hypoglycemia. It is possible to limit insulin doses and insulin-associated weight gain, however, by increasing patients' insulin sensitivity. This can accomplished by diet, exercise, and the use of insulin-sensitizing drugs such as metformin. [17] Furthermore, a systematic review of 27 studies in a subset of countries in the MENA region revealed three key areas crucial for optimal management of type 2 diabetes: glycemic control, hypertension, and dyslipidemia [26].

\section{Conclusion:-}

In our study, we report a high prevalence of overweight and obesity among diabetic patients in the western region of Saudi Arabia, with the BMI in diabetic patients taking OHG being significantly higher than that in diabetic patients not taking OHG. BMI did not significantly differ between diabetic patients taking insulin and those not taking insulin. Taken as a whole, increasing awareness regarding the risk of obesity and diabetes through targeted public education initiatives could provide an effective way to improve the modifiable risk factors for diabetes and obesity in adults in the western region of Saudi Arabia, rather than relying on screening and supplementation programs that are expensive and may not be accessible by all adults. Increasing awareness among patients with diabetes regarding the role of exogenous insulin replacement therapy in weight gain and bad prognosis if the patients has not promotes good lifestyle activities. Promotion of outdoor activities and healthy diet are necessary components for achieving optimal weight and diabetic control.

\section{References:-}

1. World Health Organization (WHO) (2006) Diabetes mellitus fact sheet no. 312. Geneva.

2. 2.Mehuys E, De Bolle L, Van Bortel L, Annemans L, Van Tongelen I, Remon JP, et al. Medication use and disease management of type 2 diabetes in Belgium. Pharm World Sci 2008; 30: 51-56.

3. Wild S, Roglic G, Green A, Sicree R, King H. Global prevalence of diabetes: estimates for the year 2000 and projections for 2030. Diabetes Care 2004; 27: 1047-1053

4. Shaw JE, Sicree RA, Zimmet PZ. Global estimates of the prevalence of diabetes for 2010 and 2030. 2010; 87: 4-14.

5. Hamadeh RR. Noncommunicable diseases among the Bahraini population: a review. East Mediterr Health $J$ 2000; 6: 1091-1097. 
6. Al-Lawati JA, Al Riyami AM, Mohammed AJ, Jousilahti P. Increasing prevalence of diabetes mellitus in Oman. Diabet Med2002; 19: 954-957.

7. Cheng YJ, Imperatore G, Geiss LS, Wang J, Saydah SH, Cowie CC, et al. Secular changes in the age-specific prevalence of diabetes among U.S. adults: 1988-2010. Diabetes Care 2013;36:2690-2696.

8. Mansour M. Al-Nozha, Mohammed A. Al-Maatouq, Yaqoub Y. Al-Mazrou, Saad S. Al-Harthi, FACHARZT, Mohammed R. Arafah, FACC, Mohamed

9. Kodama S, Horikawa C, Fujihara K, Heianza Y, Hirasawa R, Yachi Y, et al. Comparisons of the strength of associations with future type 2 diabetes risk among anthropometric obesity indicators, including waist-to-height ratio: a meta-analysis. Am J Epidemiol 2012;176: 959-969.

10. Warsy AS, El-Hazmi MA. Diabetes mellitus, hypertension and obesity-Common multi-factorial disorders in Saudis. East Mediterr Health J 1999; 5: 1236-1242.

11. al-Nuaim AR, al-Rubeaan K, al-Mazrou Y, al-Attas O, al-Daghari N, Khoja T. High prevalence of overweight and obesity in Saudi Arabia. Int J Obes Relat Metab Disord 1996; 20: 547-552.

12. al-Nuaim AA, Bamgboye EA, al-Rubeaan KA, al-Mazrou Y. Overweight and obesity in Saudi Arabian adult population, role of socio-demographic variables. J Community Health 1997; 22: 211-223.

13. Carnethon MR, De Chavez PJ, Biggs ML, Lewis CE, Pankow JS, Bertoni AG, et al. Association of weight status with mortality in adults with incident diabetes. JAMA 2012; 308: 581-590.

14. Alhyas L, McKay A, Balasanthiran A, Majeed A. Prevalences of overweight, obesity, hyperglycaemia, hypertension and dyslipidaemia in the Gulf: systematic review. JRSM Short Rep 2011; 2: 55.

15. Russell-Jones D, Khan R. Insulin-associated weight gain in diabetes - causes, effects and coping strategies. Diabetes Obes Metab 2007; 9: 799-812.

16. Alberti KG, Zimmet P, Shaw J. Metabolic syndrome - a new world-wide definition. A Consensus Statement from the International Diabetes Federation. Diabet Med 2006; 23: 469-480.

17. Khandekar S, Noeman SA, Muralidhar K, Gadallah M, Al-Sawaf KS. Central adiposity and atherogenic lipids in Saudi diabetics. Ann Saudi Med 1994;14:329-332.

18. Musaiger A, Qashqari K. The relation between dressing and obesity among women in Saudi Arabia. Arab $J$ Food Nutr 2005; 6: 292-302.

19. al-Mahroos F, al-Roomi K. Overweight and obesity in the Arabian Peninsula: an overview. J R Soc Promot Health 1999; 119: 251-253.

20. Amin TT, Al-Sultan AI, Ali A. Overweight and obesity and their association with dietary habits, and sociodemographic characteristics among male primary school children in Al-Hassa, Kingdom of Saudi Arabia. Indian J Community Med 2008; 33: 172-181.

21. Busch CP, Hegele RA. Genetic determinants of type 2 diabetes mellitus. Clin Genet 2001; 60: 243-254.

22. Dugoujon JM, Guitard E, Sénégas MT, Roth MP, Sanchez A, Barny S, et al. Genetic markers of immunoglobulins and diabetes mellitus in the multiracial population of New Caledonia. The CALDIA Study Group. Diabetes Res Clin Pract 2000; 47: 209-215.

23. Gast KB, Tjeerdema N, Stijnen T, Smit JW, Dekkers OM. Insulin Resistance and Risk of Incident Cardiovascular Events in Adults without Diabetes: Meta-Analysis. PLoS One 2012; 7: e52036.

24. Conway B, Miller RG, Costacou T, Fried L, Kelsey S, Evans RW, et al. Adiposity and mortality in type 1 diabetes. Int J Obes (Lond) 2009; 33: 796-805.

25. van Vliet M, van der Heyden JC, Diamant M, von Rosenstiel IA, Schindhelm RK, Aanstoot HJ, et al. Overweight is highly prevalent in children with type 1 diabetes and associates with cardiometabolic risk. $J$ Pediatr 2010; 156: 923-929.

26. Alhyas L, McKay A, Balasanthiran A, Majeed A. Quality of type 2 diabetes management in the states of the Co-operation Council for the Arab States of the Gulf: a systematic review. PLoS One 2011; 6: e22186. 